\title{
SHARP BOUNDS ON THE SINC FUNCTION VIA THE FOURIER SERIES METHOD
}

\begin{abstract}
GABRIEL BERCU
Abstract. In this paper we provide sharp bounds on the sinc function using the Fourier series method. Refinements of some remarkable trigonometric inequalities involving sinc function are given as well.

Mathematics subject classification (2010): 41A21, 42B05, 26D05, $26 \mathrm{D} 15$.

Keywords and phrases: Sinc function, Fourier series, trigonometric inequalities.
\end{abstract}

\section{REFERENCES}

[1] ABE U., CACCIA D., A sharpening of Jordan's inequality, Am. Math. Mon., 93 (7) (1986), pp. 568 569.

[2] Agarwal R. P., Kim Y., Sen S. K., A new refined Jordan's inequality and its applications, Math. Ineq. Appl., 12 (2) (2009), pp. 255 - 264.

[3] Boyd J. P., Chebyshev and Fourier Spectral Methods, 2nd edition, New York, NY, USA: Dover, (2000).

[4] Chen C. - P., SÁndor J., Sharp inequalities for trigonometric and hyperbolic functions, J. Math. Ineq., 9 (1) (2015), pp. $203-217$.

[5] Debnath L., Zhao C. - J, New strenghthened Jordan's inequality and its applications, Appl. Math. Lett., 16 (4) (2003), pp. $557-560$.

[6] Debnath L., Mortici C., Zhu L., Refinements of Jordan-Stečkin and Becker-Stark inequalities, Results Math., 67 (1-2) (2015), pp. $207-215$.

[7] JiAng W. - D., HUA Y., Note on Wilker's inequality and Huygens's inequality, Commun. Stud. Ineq., 13 (1) (2006), pp. 149 - 151.

[8] Kuang J. - C., Applied Inequalities, Shandong Science and Technology Press, Ji'nan City, China, 3rd edition, (2004).

[9] Kuo M. - K., Refinements of Jordan's inequality, J. Ineq. Appl., 2011( 2011):130.

[10] MALEŠEviĆ B. J., An application of $\lambda$ - method on inequalities of Shafer - Fink - type, Math. Inequal. Appl., 10 (3) (2007), pp. 529 - 534.

[11] Mitrinović D. S., Analytic Inequalities, New York, NY, USA: Springer - Verlag, (1970).

[12] Qi F., Niu D. - W., Guo B. - N., Refinements, generalizations, and applications of Jordan's inequality and related problems, J. Inequal. Appl., (2009), Article ID 271923, 52 pages, doi: $10.1155 / 2009 / 271923$.

[13] Redheffer R., Problem 5642, Am. Math. Mon. 76, (1969), pp.422.

[14] WU S. - H., SRIVASTAVA H. M., A further refinement of a Jordan type inequality and its applications, Appl. Math. Comput., 197 (2) (2008), pp. 914 - 923.

[15] Wu S. - H., Srivastava H. M., Debnath L., Some refined families of Jordan - type inequalities and their applications, Integr. Transf. Spec. Func., 19 (3 - 4) (2008), pp.183 - 193.

[16] Yue Hu L., Mortici C., A lower bound on the sinc function and its applications, Sci. World J. (2014), doi: 10.1155/2014/571218.

[17] ZHU L., Sharpening Redheffer - type inequalities for circular functions, Appl. Math. Lett., 22 (5) (2009), pp. $743-748$.

[18] ZHU L., Sun J., Six new Redheffer - type inequalities for circular and hyperbolic functions, Comput. \& Math. Appl., 56 (2) (2008), pp. $522-529$. 